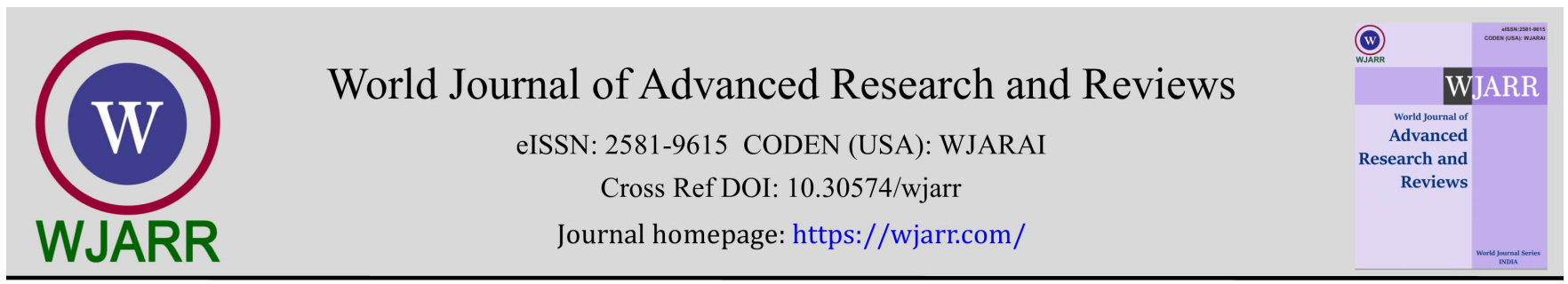

(REVIEW ARTICLE)

Check for updates

\title{
Understanding psychosis: diagnosis and clinical presentation (updates for clinicians)
}

\author{
Gayane Kirakosyan * and Alina Frolova \\ Regional Referral Psychiatric Hospital, Odesa, Odesa Region, Ukraine.
}

World Journal of Advanced Research and Reviews, 2022, 13(01), 065-071

Publication history: Received on 28 November 2021; revised on 01 January 2022; accepted on 03 January 2022

Article DOI: https://doi.org/10.30574/wjarr.2022.13.1.0759

\begin{abstract}
Psychosis is understood as the brightest manifestations of mental illness, in which the patient's mental activity does not correspond to the surrounding reality, the reflection of the real world in consciousness is sharply distorted, which manifests itself in behavioral disorders, abnormal pathological symptoms and syndromes. Psychosis is a combination of biological (genetic, neuroanatomical, neurophysiological), psychological and social factors in various proportions. Psychoses are classified according to their origin (etiology) and reasons (pathogenetic mechanisms of development) into endogenous (including endogenous psychoses include schizophrenia, schizoaffective disorder, some psychotic forms of affective disorders), organic, somatogenic, psychogenic (reactive, situational), intoxication, withdrawal and post-withdrawal. Most often, psychoses develop in the framework of so-called endogenous disorder. The concepts of psychosis and schizophrenia are often equated, which is incorrect as psychotic disorders can occur in a number of mental illnesses: Alzheimer's disease and other types of dementia, chronic alcoholism, drug addiction, epilepsy, intellectual disabilities, etc. Other types of psychosis, such as infectious, somatic and intoxication psychoses are quite often find among patients in non-psychiatric practices. This review article is a good educational material for medical and psychological practitioners whose goal is to improve knowledge and diagnostic processes of psychosis and its related disorders.
\end{abstract}

Keywords: Psychosis; Schizophrenia; Mood Disorders; Child Psychosis; Psychosis in General Practice; Depression; Bipolar Disorder; Delirium

\section{Introduction}

Psychosis refers to a state of mind in which confusion arises between the real and the unreal. Psychosis can affect all five human senses, behavior and emotions. During a period of psychosis, the mind "loses" touch with reality. A person may have experiences that confuse and frighten not only themselves, but also those around them. Symptoms of psychosis vary, but two common symptoms are hallucinations and delusions [1-3]. The hallucinated person will hear, feel, see, smell, or taste something that is not actually happening. Hallucinations, while not grounded in reality, are real to the person who experience them and can therefore be very frightening and life-threatening. Delusion is when a person firmly believes in what society usually considers to be false or not based on reality. These beliefs can be intimidating, confusing and interfering with the daily life of the person and those around them. Psychosis usually results from a combination of a person's genetics and life experiences. Stressful events, substance use or even physical health conditions (epilepsy, dementia, Parkinson's disease and even stroke) can cause psychosis in some people [4-8]. The National Institute of Mental Health reports that up to three out of every 100 people will experience episodes of psychosis in their lives [9]. Sometimes extreme experiences can cause someone a short period of psychosis that lasts only a few days and then never appear again. For others, psychosis can be a sign of a mental condition, such as schizophrenia, schizoaffective disorder, bipolar disorder, major depression or even delirium [10-15].

\footnotetext{
* Corresponding author: Gayane Kirakosyan

Regional Referral Psychiatric Hospital, Odesa, Odesa Region, Ukraine.

Copyright (C) 2022 Author(s) retain the copyright of this article. This article is published under the terms of the Creative Commons Attribution Liscense 4.0.
} 


\section{Key symptoms of psychosis}

Psychotic disorders are a very common type of psychiatry pathology. Statistical data in different regions differ from each other, which is associated with different approaches of identifying and accounting for these sometimes difficult conditions for diagnosis. On average, the frequency of psychoses is $3-5 \%$ of the population [16]. The manifestations of psychosis are truly limitless, which reflects the richness of the human psyche.

The main manifestations of psychosis are:

- Hallucinations that can be auditory, visual, olfactory, gustatory and tactile. Hallucinations can be simple (calls, noises, sounds) and complex (speech, scenes). The most common auditory hallucinations are the so-called "voices" that a person can hear coming from outside or inside the head. In most cases, voices are perceived so clearly that the patient does not have the slightest doubt about their reality. Voices can be threatening, accusing, neutral or imperative (commanding). The latter are considered the most dangerous, since patients often obey the orders of the voices and perform acts that are dangerous to themselves or others.

- Delusional ideas - judgments, inferences that do not correspond to reality, completely take possession of the patient's mind, not amenable to correction by persuasion and explanation. The content of delusional ideas can be very diverse, but most often they are: delusion of persecution (patients believe that they are being monitored, they want to kill and the conspiracies are organized), delusions of influence (from psychics, aliens, special services with the help of radiation or witchcraft), delusions of damage (add poison, steal or spoil things), hypochondriacal delusions (such patients are convinced that they suffer from some kind of disease, often severe and incurable). There are also delusions of jealousy, invention, grandiose, litigious and other origin.

- Movement disorders manifest in the form of lethargy (stupor) or excitement. With a stupor, the patient freezes in one position, becomes inactive, stops answering questions, looks at one point and refuses to eat. Patients in a state of psychomotor agitation, on the contrary, are all the time in motion, talking incessantly, sometimes grimacing, mimicking, being extremely inappropriate, aggressive and impulsive.

- Mood disorders manifested by depressive or manic states. Depression is characterized, first of all, by a decreased mood, melancholy, depression, motor and intellectual inhibition, the disappearance of desires and impulses, a decrease in energy, a pessimistic judgement of the past, present and future, ideas of self-accusation and thoughts of suicide [14-15, 17-19]. The manic state manifests itself in an unreasonably elevated mood, acceleration of thinking and motor activity, overestimation of the capabilities of one's own personality with the construction of unreal, sometimes fantastic plans and projects, the disappearance of the need for sleep and disinhibition of drives (alcohol and drug abuse and promiscuous sex) [11-12].

Often, patients become sluggish, less motivated and passive. Usually, there is a decrease in energy tone, the disappearance of desires, motivations, aspirations, increasing emotional dullness, isolation from others, unwillingness to communicate and enter into any social contacts. Often they lose their inherent responsiveness, sincerity, sense of tact and appear irritable, rude and aggressive. In addition, patients develop mental dysfunctions and become unfocused, rigid and meaningless. Also these patients can lose their previous work skills. During psychosis, patients lose their daily skills, they become neglected and this negatively affects their health (including condition of skin, hair and dental health) [20-21].

In order to formulate all of the above in a more understandable way, clinical presentation of psychosis can be embodied as follows. Psychosis can manifest itself in hallucinatory, delusional and emotional disorders and in their combination. Hallucinatory disorders present when affected patient talks to an invisible interlocutor, abrupt silence and listens to something invisible to others that can be a sign of hallucinations. Hallucinations can be auditory, visual, gustatory, tactile and olfactory. Delusions are ideas, judgments, inferences that take over a person's mind and disabling critical thinking; in this state a person cannot explain, prove or persuade. The most common are bizarre and non-bizarre delusions that can be persecutory, grandiose, jealousy, religious, hypochondriac and nihilistic. Emotional disorders are characterized by nervousness, anxiety, hypersensitivity, eating or sleeping disorders, lack of interest and energy decline. Changes of sensations include mood swings, distortions of feelings, fears and depression. Additionally, psychosis can affect ability to work and behavior in society. It negatively impacts attention, causes mistrust, lack of resilience to stress, communication problems, isolation, self-care and abrupt change of interests. Changes in perception and experience provokes distorted perception of color, sound, smell and feelings of general distrust. For members of the family whose relative suffers from mental disorder, information about the initial manifestations of psychosis or about the symptoms of an advanced stage of the disorder may be recommended. It may be also helpful to know the recommendations on some rules of behavior and communication with a person who is in a sick state. In real life, it is often difficult to 
immediately understand what is happening to their loved one, especially if they are frightened, suspicious, distrustful and does not express any complaints. In such cases, only indirect manifestations of mental disorders can be noticed. Psychosis can have a complex structure and can be atypical.

Commonly to all psychoses, patients are able to stay in a state of altered consciousness, accompanied by manifestations of delusional disorders and hallucinations. A characteristic feature is the inadequacy of behavior, which is accompanied by inadequate statements and expressions of emotions, due to the loss of reality of perception of the surrounding reality when the patient is in a situation that most people perceive as not arousing fear. The manifestations of this disorder are endless and the same clinical features can be caused by a variety of etiological factors.

\section{Understanding the development of psychosis}

Psychiatrists recognize the multifactorial system of mechanisms that gives rise to psychotic disorders. This model assumes that the same type of psychosis can start in different people for different reasons [22-23]. One of these systems is the model conventionally called "stress - vulnerability". This theory is based on the following: there are certain genetic factors that predispose to the formation of psychotic disorders in an individual. The presence of such tendency to psychosis, coupled with a specific characterological portrait of the personality, is the basis for a high susceptibility of a person to the effects of stress factors, both a positive spectrum and a negative color. At certain stages of life, for example: during puberty or during pregnancy, the subject becomes especially vulnerable to the onset of traumatic events due to its tendency to excessive mental reactions and inability to withstand stress. It is this point that is the countdown for the formation and the onset of psychosis. At the same time, not always factors that have a protective effect (for example: a person's financial stability and a happy and stable marriage) can counteract traumatic circumstances. In some situations, when the intensity of stressors is too high, such protective factors only postpone the moment of development of psychosis and mitigate the severity of the symptoms. If we consider each of the versions proposed by scientists separately, one should highlight the most proven theories describing the causes of the development of psychosis.

Psychosis occurs due to problems in the functioning of neurons. Due to the violation of bonds in the molecules, they do not receive nutrition and they are deficient in oxygen. This leads to the fact that neurons cannot transmit nerve impulses and as a result multiple dysfunctions occur in the central nervous system. The type of psychosis that occurs depends on which part of the brain suffered from the hunger strike. There are 3 causes of this dysfunction:

- $\quad$ Endogenous that associated with internal processes. It can be endocrine and neurological disorders, bronchial asthma, vitamin B deficiency, conditions accompanied by acute pain (ulcer, heart attack, sarcoidosis), electrolyte imbalance, systemic diseases, genetic predisposition, age, etc.

- Exogenous, or external: stress, intoxication with alcohol and other substances, drugs, the consequences of infectious processes, psychological trauma, etc.

- $\quad$ Organic, when the causes of psychosis are changes in the brain: tumors, trauma or hemorrhage.

- Schizophrenia is a specific mental health condition in which symptoms of psychosis appear. These symptoms can come and go and are often helped by medications. In addition to hallucinations and illusions, people living with schizophrenia may also experience decreased interest and motivation for action, difficulty expressing or interpreting emotions, or withdrawal from social activities and relationships. People living with schizophrenia also experience cognitive symptoms such as poor decision-making ability, focusing on tasks and using information immediately after learning it. Symptoms of schizophrenia usually develop between the ages of 16 and 30, but can even be presented at childhood [23]. The cause of schizophrenia is unknown, but researchers believe that genes and their interactions with the human environment do play a role in the development of the disease, as well as in various chemical balances in the brain.

Another cause of psychosis is drug and alcohol use, called substance-use disorder. This condition causes symptoms such as hallucinations and delusions. In most cases, these symptoms are short-lived, lasting only a few hours or days. In rare cases, heavy and prolonged drug use can cause psychosis that lasts for months or years, long after the drug has left the body [24]. Similar to substance use disorder, non-chemical addictions (e.g., pathological gambling) can be associated with psychotic presentation [25].

Special attention should be given to depression and its ability of provoking psychosis. False inferences that cannot be dissuaded, ideas of guilt, ideas of worthlessness, ideas of referral can be a part of psychosis associated with depression [26-27]. The judgments are erroneous, but they completely embrace the patient's mind. In this case, the behavior is entirely determined by the crooked logic of disturbed thinking. Under the influence of a painful condition, the affected 
person may refuse to communicate, stop eating, take care of themselves and commit suicide. With depressive psychosis, the speed of the flow of thoughts is disturbed: most often, associative processes slow down, but there are cases when thoughts are accelerated, which becomes noticeable by accelerated speech.

Neurodevelopmental disorders, those diagnosed mainly in childhood, can also be associated with the onset of psychosis. These disorders include autism spectrum disorder and attention deficit hyperactivity disorder (ADHD) [28-30]. ADHD is a risk factor for developing psychosis in adulthood. Most often it is provoked by drugs from the group of central stimulants. As indirect dopamine agonists, these drugs increase the concentration of neurotransmitters in the intercellular space in the prefrontal cortex, which may be the cause of psychotic symptoms. Most people with autism have one or more mental disorders. In the absence of treatment, mental disorders can lead to a dramatic worsening of behavioral problems in autism. However, due to similar symptoms, diagnosing autism can be very difficult. For example, avoiding social contact in depression or psychosis is difficult to distinguish from social interactions associated with autism. In addition, people with autism may find it difficult to identify or express their emotions and other inner experiences.

\section{Different types of psychosis}

As previously mentioned, types of psychosis are divided into endogenous, exogenous and organic. Such a division is conditional as psychosis e.g., resulting from traumatic brain injury can develop further against the background of mental trauma or under the influence of toxic agents.

Depressive psychosis lasts from 3 months to a year and is associated with brain pathology, while depression begins slowly and imperceptibly. The main signs of this type of psychosis include constantly low mood, physical and mental retardation. Some psychiatrists believe that this form of psychosis is characteristic of highly moral people. The patient thinks only of themselves, blames themselves, looks for their mistakes and shortcomings. A person's thoughts are centered around their personality and their mistakes. Such person has no doubts that nothing good in their life has been and will not be, in such a state they can finish their life. With depressive psychosis, the state is worst in the morning and in the evening it rises.

Mass (psychogenic) psychosis is a crowd epidemic based on suggestibility and imitation psychosis. The disorder affects a group of people, causing them to become obsessed. The most popular mass psychoses these days are considered: virus phobia, computer gambling addiction, chat mania and aerophobia. Approximately the same is the induced form of the disease, the only difference is that here one person, usually mentally unhealthy, purposefully instills delusional ideas to others.

Deferred psychosis develops due to long-term use of anticholinergic drugs or antipsychotics. It can also form against the background of its cancellation.

Induced psychosis occurs when a delusional system develops in a patient as a result of a close relationship with another person who has previously been found to have a similar delusional system [2-3]. This disorder used to be called "paranoid disorder shared with another person" or "folie a deux". However, since the pathogenesis and course of this disorder was found to be different from other delusional disorders, the common (with the other person) delusional disorder was renamed. Induced psychosis is a rare disorder and usually affects two people. Cases in which more than two persons develop induced psychosis are called folie a trois or a quatre, etc. One case involving a whole family called folice a douse, where up to 12 people could be included. Other names that are also sometimes used are "double insanity" and "psychosis of association".

Postpartum psychosis rarely develops, the first symptoms of the disease form on average 5 weeks after childbirth. This mental disorder manifests itself as hallucinations, paranoia, delusions and a desire to harm the child or oneself. The disorder can also begin during pregnancy, for example, due to inattention, misunderstanding and abuse of a beloved one.

Amphetamine psychosis is caused by amphetamine and its derivatives, when taken it regularly or at high dosages, causes constant anxiety and tension, delusions, visual and auditory hallucinations.

Late onset psychosis occurs in the elderly, more often in women. Depression, melancholy, hallucinosis and paranoids of late age may develop. The disorder is more common in patients living in nursing homes. 
In vascular psychosis, the source of disease lies in vascular disorders of the brain (hypertension, atherosclerosis, thrombosis and hypotension). At the same time, patients complain of ringing in the ears, morning headache in the occipital region, twitching of the facial muscles and numbness in the chin, cheeks, and nose.

Paranoid psychosis is more severe than paranoia, but more favorable than delusional disorder. In this case, mood disorders are accompanied by the idea of persecution and pseudohallucinations are possible.

Intoxication psychoses develop as a result of the toxic effect on the body of industrial and food poisons, medications, pesticides, alcohol, etc. In this case, delirium is observed, turning into stupor and coma. In the future, memory is impaired, intellectual abilities decrease and dementia develops.

Postoperative psychosis appears in patients after surgery, mainly against the background of intoxication. At the same time, the person is restless, trying to escape, jump out of the window, delirious.

Finally, epileptic psychosis often occurs as a complication of epilepsy, especially in childhood and adolescence. It usually goes away quickly, but at a later stage it can last for a year.

\section{Conclusion}

Psychosis is a mental illness during which an affected person cannot adequately behave, does not perceive the world around them adequately and does not react to it appropriately. At the initial stages, psychosis can be recognized by changes in behavior, emotional disturbance and a person may develop atypical reactions. Such affected people cannot control their feelings and expresses emotions inappropriate to different situations. The onset of psychosis may indicate the development of schizophrenia, delirium tremens, dementia and other serious mental and physical illnesses. It is crucial task for every medical practitioner to be able to recognize psychosis during performance of their professional duties as it may help to prevent its negative outcome.

\section{Compliance with ethical standards}

\section{Acknowledgments}

We thank our supervisors for their support during the preparation process of this manuscript.

\section{Disclosure of conflict of interest}

The authors declare that there is no conflict of interest.

\section{Statement of ethical approval}

No ethical approval for this type of manuscript is needed.

\section{Statement of informed consent}

This is a review article and therefore the informed consent is not required.

\section{References}

[1] Cuesta MJ, de Jalón EG, Campos MS, Ibáñez B, Sánchez-Torres AM, Peralta V. Duration of untreated negative and positive symptoms of psychosis and cognitive impairment in first episode psychosis. Schizophrenia research, 2012; 141(2-3): 222-227.

[2] Tsarkov A, Patrick M, Petro P. Uncommon presentation: Folie à deux (Case study). World Journal of Advanced Research and Reviews (WJARR). 2020; 6: 43-49.

[3] Tsarkov A, Msoni P, Petlovanyi P. Induced Delusional Disorder: A Case Report. British Journal of Medical and Health Research. 2018; 12-22.

[4] Joyce EM. Organic psychosis: the pathobiology and treatment of delusions. CNS neuroscience \& therapeutics. 2018; 24(7): 598-603. 
[5] Tsarkov A, Petlovanyi P. Neuropsychiatric aspects of a common problem: stroke. European Journal of Medical and Health Sciences (EJMED). 2019; 1(3): 1-6.

[6] Lebedyn Z. Diagnostic Procedure and Therapeutic Approaches in Nonconvulsive Status Epilepticus (NCSE). Origins (toxic/dysmetabolic, or of mixed etiology). 2020; 15: 18.

[7] Tsarkov A, Petlovanyi P. Use of pramipexole in neuropsychiatry. World Journal of Advanced Research and Reviews (WJARR). 2020; 7(2): 82-88.

[8] Tsarkov A, Petlovanyi P, Paul R, Prashar L. Modern approach to the treatment of Parkinson's disease: the role of pramipexole in the correction of motor and non-motor disorders. British Journal of Medical and Health Research (BJMHR). 2017; 4(2): 63-71.

[9] The National Institute of Mental Health. Fact Sheet: First Episode Psychosis. Recovery After an Initial Schizophrenia Episode (RAISE). 2021.

[10] Kirakosyan G, Frolova A. Delirium (Clinical Presentation, Diagnosis and Treatment): Updates for Neurologists and Psychiatrists. 2020.

[11] Tsarkov A, Petlovanyi P. Bipolar Disorder in Child Psychiatric Practice: A Case Report. Medical Journal of Zambia. 2016; 43(1): 41-46.

[12] Tsarkov A, Petlovanyi P. Bipolar disorder in child psychiatric practice. The African Digital Health Library (ADHL). 2016.

[13] Upthegrove R, Ross K, Brunet K, McCollum R, Jones L. Depression in first episode psychosis: the role of subordination and shame. Psychiatry research. 2014; 217(3): 177-184.

[14] Tsarkov A, Petlovanyi P. Depressive Disorder in Child Psychiatric Practice: A Case Report. The Health Press (Zambia). 2017; 9-16.

[15] Tsarkov A, Petlovanyi P. Depressive Disorder in Child Psychiatric Practice: A.

[16] Wüsten C, Schlier B, Jaya E, Fonseca-Pedrero E, Peters E, Verdoux H, Lincoln T. Psychotic experiences and related distress: a cross-national comparison and network analysis based on 7141 participants from 13 countries. Schizophrenia bulletin. 2018; 44(6): 1185-1194.

[17] Tsarkov A, Petlovanyi P. The Role of Lamotrigine in the Treatment of Bipolar Depression. Imperial Journal of Interdisciplinary Research (IJIR). 2017; 3(8): 131-134.

[18] Lebedyn, Z. Recommendations For The Management Of Patients With Depression In General Clinical Practice. European Journal of Medical and Health Sciences. 2020; 2(4).

[19] Konyushok M. Why Neurologists Should Remember About Antidepressants. Bipolar disorder. 2020; 2(4).

[20] Sharp I, Kobak K, Osman D. The use of videoconferencing with patients with psychosis: a review of the literature. Annals of general psychiatry. 2011; 10(1): 1-11.

[21] Phiri C, Tsarkov A, Petlovanyi P, Lingenda. Factors Contributing To Oral Diseases and Treatment Needs amongst Mental Patients at Chainama Hills College Hospital, Lusaka, Zambia. Imperial Journal of Interdisciplinary Research (IJIR). 2017; 3(11): 495-504.

[22] Bora E, Pantelis C. Theory of mind impairments in first-episode psychosis, individuals at ultra-high risk for psychosis and in first-degree relatives of schizophrenia: systematic review and meta-analysis. Schizophrenia research. 2013; 144(1-3): 31-36.

[23] Petlovanyi P, Tsarkov A. Child Schizophrenia: Theory and Practice. European Journal of Medical and Health Sciences (EJMED). 2020; 2(1): 1-5.

[24] Wisdom JP, Manuel JI, Drake RE. Substance use disorder among people with first-episode psychosis: a systematic review of course and treatment. Psychiatric Services. 2011; 62(9): 1007-1012.

[25] Tsarkov A, Petlovanyi P. Pathological Gambling: The Old Problem of the Modern World. Imperial Journal of Interdisciplinary Research (IJIR). 2017; 3(8): 216-221.

[26] Lebedyn, Z. The Use of Antidepressants by General Practitioners and Psychiatrists (Personal Experience). International Journal of Science and Research (IJSR). 2019.

[27] Lebedyn, Z. Antidepressants In General Practice And Psychiatry. European Journal of Medical and Health Sciences. 2020; 2(3). 
[28] Petlovanyi P, Tsarkov A. Practical guide and some recommendations for the diagnosis and management of Attention deficit hyperactivity disorder (ADHD). World Journal of Advanced Research and Reviews (WJARR). 2020; 6(3): 257-261.

[29] Tsarkov A, Petlovanyi P. Omega-3 Fatty Acids as an Alternative Treatment for Children with Attention Deficit Hyperactivity Disorder. Imperial Journal of Interdisciplinary Research (IJIR). 2017; 3(2): 1378-1380.

[30] Konyushok, M. Autism Spectrum Disorder in Adult Patients: Clinical Presentation And Management. Psychopathology. 2020; 2(4). 